Mon. Not. R. Astron. Soc. 000, 000-000 (0000) Printed 10 May $2017 \quad$ (MN LATEX style file v1.4)

\title{
First hard X-ray detection and broad band X-ray study of the unidentified transient AX J1949.8+2534
}

\author{
V. Sguera ${ }^{1}$, L. Sidoli ${ }^{2}$, A. Paizis ${ }^{2}$, N. Masetti ${ }^{1}$, A. J. Bird ${ }^{3}$, A. Bazzano $^{4}$ \\ 1 INAF, Istituto di Astrofisica Spaziale e Fisica Cosmica, Via Gobetti 101, I-40129 Bologna, Italy \\ 2 INAF, Istituto di Astrofisica Spaziale e Fisica Cosmica, Via E. Bassini 15, I-20133 Milano, Italy \\ 3 School of Physics and Astronomy, University of Southampton, University Road, Southampton, SO17 1BJ, UK \\ ${ }^{4}$ INAF, Istituto di Astrofisica e Planetologia Spaziali, Via Fosso del Cavaliere 100, I-00133 Roma, Italy
}

Accepted 2017 May 05. In original form 2017 March 01

\begin{abstract}
We present the results from INTEGRAL and Swift/XRT observations of the hitherto poorly studied unidentified X-ray transient AX J1949.8+2534, and on archival multiwavelength observations of field objects. Bright hard X-ray outbursts have been discovered above $20 \mathrm{keV}$ for the first time, the measured duty cycle and dynamic range are of the order of $\sim 4 \%$ and $\geq 630$, respectively. The source was also detected during a low soft X-ray state $\left(\sim 2 \times 10^{-12} \mathrm{erg} \mathrm{cm}^{-2} \mathrm{~s}^{-1}\right)$ thanks to a Swift/XRT followup, which allowed for the first time to perform a soft X-ray spectral analysis as well as significantly improve the source positional uncertainty from arcminute to arcsecond size. From archival near-infrared data, we pinpointed two bright objects as most likely counterparts whose photometric properties are compatible with an early type spectral nature. This strongly supports a High Mass X-ray Binary (HMXB) scenario for AX J1949.8+2534, specifically a Supergiant Fast X-ray Transient (more likely) or alternatively a Be HMXB.
\end{abstract}

Key words: X-rays: binaries - X-rays: individual: AX J1949.8-2534

\section{INTRODUCTION}

AX J1949.8+2534 is a hitherto poorly studied unidentified X-ray source. It was discovered during the $A S C A$ Galactic Plane Survey (Sugizaki et al. 2001) whose observations were carried out from March 1996 to April 1999, covering the inner Galactic disk at $|l| \leq 45^{\circ}$ and $|b| \leq 0^{\circ} .4$. The source was detected with a significance of $3.6 \sigma$ and $21.6 \sigma$ in the energy bands $0.7-2 \mathrm{keV}$ and $2-10 \mathrm{keV}$, respectively. No spectral information is available, the reported $2-10 \mathrm{keV} A S C A$ count rate (Sugizaki et al. 2001) converts into an absorbed X-ray flux of $\sim 8 \times 10^{-12} \mathrm{erg} \mathrm{cm}^{-2} \mathrm{~s}^{-1}\left(\sim 6 \times 10^{-12} \mathrm{erg} \mathrm{cm}^{-2} \mathrm{~s}^{-1}\right)$ if we assume a power law spectral shape with $\Gamma=1(\Gamma=2)$. The uncertainty on the source position is $\sim 1^{\prime}$ as is typical for $A S C A \mathrm{X}$-ray sources, this prohibits the search for counterparts at lower energies (i.e. optical and infrared) which is essential to firmly identify the nature of the source.

For many years, the $A S C A$ detection represented the only information available in the literature. Interestingly, Sguera et al. (2015) have recently reported the first hard Xray detection of AX J1949.8+2534 above $20 \mathrm{keV}$. Their communication contained only very short information about the hard X-ray activity detected by INTEGRAL, as obtained from analysis of near real time data pertaining to public observations of the Cygnus region.

Here we present the results of a more detailed spectral and temporal analysis of the consolidated INTEGRAL data pertaining to the outburst reported by Sguera et al. (2015), together with the investigation of additional archival $I N$ TEGRAL data, with the aim of finding further hard X-ray activity from the source. We also report a Target of Opportunity (ToO) observation made with the Swift satellite in order to refine the error circle to arcsecond size as well as to characterize for the first time the spectral shape in the soft X-ray band.

\section{INTEGRAL}

\subsection{Data analysis}

For our study, we used data collected with the ISGRI detector (Lebrun et al. 2003) which is the lower energy layer of the IBIS coded mask telescope (Ubertini et al. 2003) onboard INTEGRAL (Winkler et al. 2003). The reduction and analysis of the data have been performed by using the Offline Scientific Analysis (OSA) version 10.1. For IBIS/ISGRI spectral analysis we used the standard 13 energy channel 
Table 1. Log of IBIS/ISGRI observations used for our study on AX J1949.8+2534. Orbits in boldface contain the IBIS/ISGRI detections reported in section 2.2 . $†$ Detection originally reported by Sguera et al. (2015).

\begin{tabular}{lccc}
\hline \hline $\begin{array}{l}\text { Telescope } \\
\text { Orbit }\end{array}$ & Date & $\begin{array}{c}\text { Observation } \\
\text { Target }\end{array}$ & $\begin{array}{c}\text { Exposure } \\
(\mathrm{ks})\end{array}$ \\
\hline 1600 & 18-20 Oct 2015 & Cyg X-1 & $\sim 22$ \\
1601 & 22-23 Oct 2015 & Cyg X-1 & $\sim 8$ \\
1602 & 23-25 Oct 2015 & GPS+Cyg X-1 & $\sim 14$ \\
1603 & 26-28 Oct 2015 & GPS+Cyg X-1 & $\sim 7$ \\
$\mathbf{1 6 0 5} \dagger$ & 31 Oct - 02 Nov 2015 & GPS+Cyg X-1 & $\sim 16$ \\
1606 & 03 Nov 2015 & GPS & $\sim 3.5$ \\
1607 & 05-07 Nov 2015 & GPS+Cyg X-1 & $\sim 22$ \\
1609 & 12-13 Nov 2015 & Cyg X-1 & $\sim 6$ \\
1610 & 14-15 Nov 2015 & Cyg X-1 & $\sim 12$ \\
1611 & 18 Nov 2015 & GPS & $\sim 3.5$ \\
1613 & 23 Nov 2015 & GPS & $\sim 4$ \\
1614 & 24-26 Nov 2015 & GPS+Cyg X-1 & $\sim 20$ \\
1616 & 01 Dec 2015 & GPS+Cyg X-1 & $\sim 7$ \\
1618 & 05 Dec 2015 & GPS & $\sim 4$ \\
1619 & 09 Dec 2015 & GPS & $\sim 4$ \\
1621 & 13-15 Dec 2015 & Cyg X-1 & $\sim 15$ \\
1624 & 21-23 Dec 2015 & GPS+Cyg X-1 & $\sim 18$ \\
1626 & 26-28 Dec 2015 & ToO V404 Cyg & $\sim 17$ \\
1627 & 29-31 Dec 2015 & ToO V404 Cyg & $\sim 16$ \\
$\mathbf{1 6 2 8}$ & 31 Dec - 02 Jan 2016 & ToO V404 Cyg & $\sim 18$ \\
$\mathbf{1 6 2 9}$ & 03-05 Jan 2016 & ToO V404 Cyg & $\sim 19$ \\
\hline & & & $\sim 255$ \\
\hline \hline
\end{tabular}

response matrix available at the INTEGRAL Science Data Centre (ISDC). INTEGRAL observations during each orbit ("revolution", lasting $\sim 2.6$ days or $170 \mathrm{ks}$ ) are divided into short pointings (Science Window, ScW) having a typical duration of $\sim 2,000 \mathrm{~s}$.

Our total data set consists of all public observations which covered the Cygnus region (i.e. Galactic Plane Scans GPS, ToO observations of V404 Cyg and targeted observations of $\mathrm{Cyg} \mathrm{X}-1$ ) immediately before and after the hard X-ray detection of AX J1949.8+2534 reported by Sguera et al. (2015). The corresponding data set amounts to a total exposure of $\sim 255 \mathrm{ks}$ (see Table 1 for details).

We performed an analysis of the full data set on two different timescales, i.e. at $\mathrm{ScW}$ level as well as at revolution level, in order to search for newly discovered X-ray activity from the source detected with a significance equal or greater than at least $5 \sigma$ and $7 \sigma$, respectively. Such detection thresholds are essential to avoid false detections/excesses caused by background noise (e.g. Bird et al. 2016). The search was initially performed in the energy band $22-60 \mathrm{keV}$; this choice takes into account the evolution of the IBIS/ISGRI energy threshold that occurred from revolution number $\sim$ 900 on. When a significant detection was found, we have also checked the detection at higher energies (i.e. 60-100 $\mathrm{keV}$ ) or in other different ranges (i.e. 22-30, 30-60 and 22$40 \mathrm{keV}$ ). We note that the sensitivity limit for a persistent source detected at $5 \sigma$ level $(22-60 \mathrm{keV})$ in only one ScW of about 2,000 s duration is $\sim 18 \mathrm{mCrab}$ (Krivonos et al. 2010).

The X-ray monitor JEM-X (Lund et al. 2003) makes observations simultaneously with IBIS/ISGRI, although
Table 2. List of INTEGRAL orbits during which significant source detections (i.e. $\geq 7 \sigma$ ) were obtained in the energy band $22-60 \mathrm{keV}$. The table also lists the date of the beginning of the outburst, significance detection over the entire activity, average flux and approximate duration.

\begin{tabular}{ccccc}
\hline \hline $\begin{array}{c}\text { Orbit } \\
(\text { n. })\end{array}$ & $\begin{array}{c}\text { Date } \\
(\mathrm{MJD})\end{array}$ & $\begin{array}{c}\text { Significance } \\
(\sigma)\end{array}$ & $\begin{array}{c}\text { Flux } \\
(\mathrm{mCrab})\end{array}$ & $\begin{array}{c}\text { Duration } \\
(\text { days })\end{array}$ \\
\hline 1605 & 57327.35 & 7.1 & $10.0 \pm 1.4$ & $\sim 1.5$ \\
1628 & 57387.65 & 7.1 & $10.6 \pm 1.5$ & $\sim 2$ \\
1629 & 57390.31 & 7.3 & $9.5 \pm 1.3$ & $\sim 2$ \\
\hline \hline
\end{tabular}

with a much smaller Field of View (FoV), providing images in the softer energy band $3-35 \mathrm{keV}$. JEM-X data were analyzed when the source was in its FoV in order to search for X-ray activity in both the energy bands $3-10 \mathrm{keV}$ and 10-20 keV.

Throughout the paper, the spectral analysis was performed using XSPEC version 12.9.0 and, unless stated otherwise, errors are quoted at the 90 per cent confidence level for one single parameter of interest.

\subsection{Results}

We report on newly discovered hard X-ray transient activity from AX J1949.8+2534, the first ever above $20 \mathrm{keV}$. Hard Xray detections with IBIS/ISGRI were obtained by analyzing data in revolutions number 1605, 1628 and 1629. Table 2 reports a summary of the outbursts main characteristics.

\subsubsection{IBIS/ISGRI detection in revolution 1605}

Firstly, we note that AX J1949.8+2534 was not detected in any single revolution from n. 1600 to n. 1603, nor in their mosaic for a total on source exposure of $\sim 50 \mathrm{ks}$. As a result, we inferred a $3 \sigma$ upper limit of $\sim 2$ mCrab $(22-60 \mathrm{keV})$.

Conversely, AX J1949.8+2534 was detected with a significance of $7.1 \sigma(22-60 \mathrm{keV})$ during revolution $1605(\sim 14$ ks on-source exposure) from 2015 Nov 01 08:28 (UTC) to 2015 Nov 02 15:48 (UTC). No detection was obtained in the energy band 60-100 keV. The measured average $22-60 \mathrm{keV}$ flux is $10.0 \pm 1.4 \mathrm{mCrab}$ ( or $\sim 1.1 \times 10^{-10} \mathrm{erg} \mathrm{cm}^{-2} \mathrm{~s}^{-1}$ ). The source was never significantly detected at ScW level (i.e. $\geq 5 \sigma$ ) at any point of the observation, indicating that no major flaring activity took place on short timescales (i.e. $\sim 2,000 \mathrm{~s})$.

The extracted IBIS/ISGRI spectrum was fitted by a power law with $\Gamma=2.9 \pm 0.8\left(\chi_{\nu}^{2}=1.7,4\right.$ d.o.f. $)$ or alternatively by a thermal bremsstrahlung with $\mathrm{kT}=24_{-9}^{+22} \mathrm{keV}\left(\chi_{\nu}^{2}=1.5\right.$, 4 d.o.f.). The best fit was achieved by using a black body model $\left(\chi_{\nu}^{2}=1.15,4\right.$ d.o.f. $)$ with $\mathrm{kT}=7.9_{-1.9}^{+1.8} \mathrm{keV}$. The average $18-60 \mathrm{keV}(20-40 \mathrm{keV})$ flux is $1.1 \times 10^{-10} \mathrm{erg} \mathrm{cm}^{-2} \mathrm{~s}^{-1}$ $\left(7.8 \times 10^{-11} \mathrm{erg} \mathrm{cm}^{-2} \mathrm{~s}^{-1}\right)$. Fig. 1 shows the black body data-to-model fit with the corresponding residuals.

The source was also in the JEM-X FoV during this observation, however in the combined JEM-X1+JEM-X2 mosaic it was not detected in both bands $3-10 \mathrm{keV}$ and 10 $20 \mathrm{keV}$ (on-source exposure of $\sim 4.8 \mathrm{ks}$ ). The inferred $3 \sigma$ upper limit $(3-10 \mathrm{keV})$ is of the order of $\sim 2 \mathrm{mCrab}$ or $4.4 \times 10^{-11} \mathrm{erg} \mathrm{cm}^{-2} \mathrm{~s}^{-1}$. 


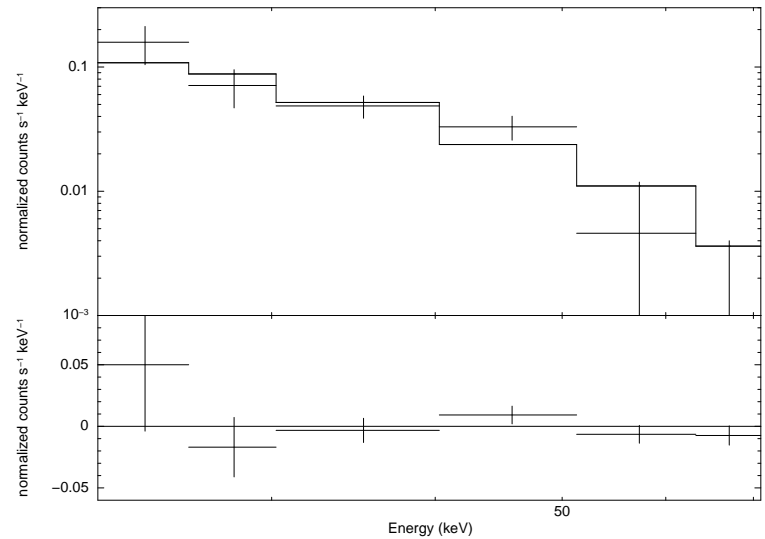

Figure 1. IBIS/ISGRI spectrum of AX J1949.8+2534 (during revolution n. 1605) best fitted by a black body. The lower panel shows the residuals from the fit.

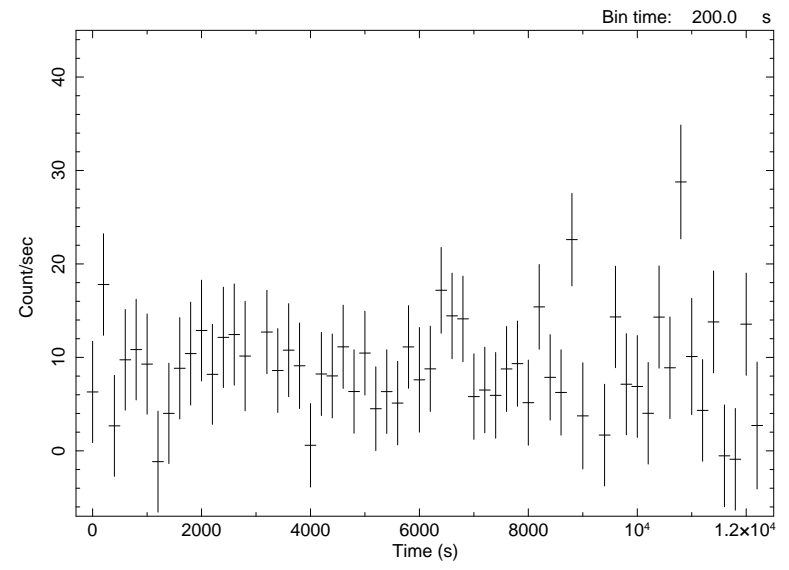

Start Time 17390 23:05:40:187 Stop Time 17391 2:29:00:187

Figure 2. IBIS/ISGRI light curve $(22-60 \mathrm{keV}, 200 \mathrm{~s}$ bin time) of AX J1949.8+2534 extracted from the four consecutive ScWs n. 20 to 23 in revolution 1629 .

AX J1949.8+2534 was not detected in any single revolution after n. 1605 (from n. 1606 to n. 1627), nor in their mosaic for a total on source exposure of $\sim 150 \mathrm{ks}$. We inferred a $3 \sigma$ upper limit of $\sim 1.2 \mathrm{mCrab}(22-60 \mathrm{keV})$. This, combined with the other upper limit from revolutions n. 1600 to 1603, allows us to confidently constrain the duration of the transient hard X-ray activity detected in revolution $\mathrm{n} .1605$ to no longer than $\sim 1.5$ days.

\subsubsection{IBIS/ISGRI detection in revolutions 1628 and 1629}

Renewed hard X-ray activity from AX J1949.8+2534 was detected again by IBIS/ISGRI towards the end of Dec 2015. In fact, the source was detected in the energy band 22 $60 \mathrm{keV}$ in both revolutions n. $1628(\sim 7.1 \sigma, \sim 18 \mathrm{ks}$ onsource exposure, $)$ and n. $1629(\sim 7.3 \sigma, \sim 19 \mathrm{ks}$ on-source exposure), spanning the time range from 2015 Dec 31 15:40 (UTC) to 2016 Jan 05 10:37 (UTC). The source showed no sign of flux variation on revolution timescale since the 22

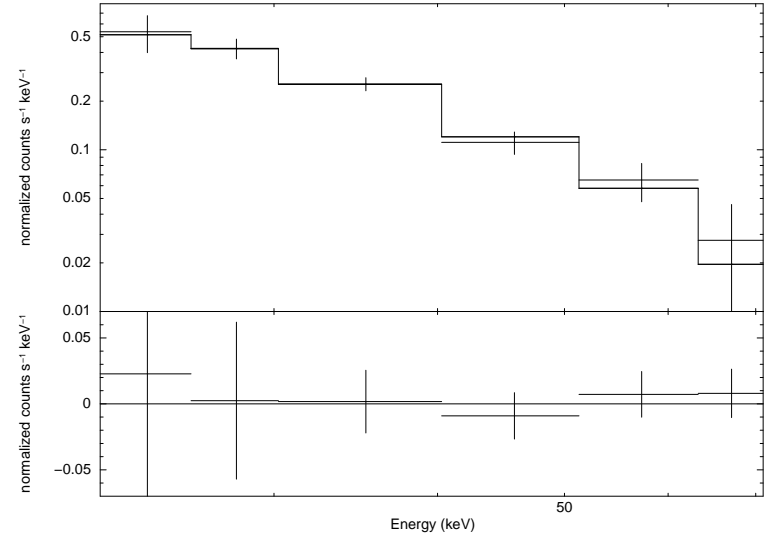

Figure 3. IBIS/ISGRI spectrum of AX J1949.8+2534 (during revolution 1629) fitted by a black body. The lower panel shows the residuals from the fit.

$60 \mathrm{keV}$ measured average fluxes are fully consistent with each other within their uncertainties $(10.6 \pm 1.5 \mathrm{mCrab}$ and $9.5 \pm 1.3 \mathrm{mCrab}$, respectively). Unfortunately, the source was not in the IBIS/ISGRI FoV again throughout revolutions after n. 1629 so we cannot constrain the duration of this latest transient hard X-ray activity, we can only infer a lower limit of $\sim 4$ days.

We stacked the data for revolutions n. 1628 and 1629 with the aim of increasing the statistics of the detection. AX J1949.8+2534 was detected in the mosaic with a significance of $9.8 \sigma(22-60 \mathrm{keV})$ for a total on-source exposure of $\sim 37 \mathrm{ks}$. No detection was obtained in the higher energy band $60-100 \mathrm{keV}$. The $22-60 \mathrm{keV}$ measured average flux is $9.30 \pm 0.95 \mathrm{mCrab}$ which is fully compatible within the errors with that measured during the hard X-ray activity detected two months earlier during revolution $\mathrm{n}$. 1605. The source was never in the JEM-X FoV during these latest observations.

We performed an investigation at ScW level of both revolutions n. 1628 and 1629 with the aim of searching for possible flaring activity on short timescales (i.e. $\sim 2,000$ s). Interestingly, we found that the source was occasionally bright enough to be significantly detected even at ScW level during the course of the observations. Table 3 lists the single ScWs during which significant detections (i.e. $\geq 5 \sigma$ ) were achieved. In particular, we focussed our attention on the four consecutive ScWs from n. 20 to 23 in revolution n. 1629 (they span a continuous temporal range of $\sim 3.5$ hours) in order to extract an IBIS/ISGRI light curve with a fine temporal bin of $200 \mathrm{~s}$. As it can be seen from Fig. 2, AX J1949.8+2534 mainly shows an enhanced and rather constant flux with no major sign of flares. Only towards the end of the light curve is there sign of possibly a couple of short flares on $\sim 200 \mathrm{~s}$ timescale, the strongest one (having a significance of $4.7 \sigma$ ) reached a peak-flux of $180 \pm 38 \mathrm{mCrab}$ or $(2 \pm 0.4) \times 10^{-9} \mathrm{erg} \mathrm{cm}^{-2} \mathrm{~s}^{-1}(22-60 \mathrm{keV})$. To establish if the source statistically varied during the entire light curve, we fitted it with a constant and applied the $\chi^{2}$ test. It was found a chance probability of 0.4 that this results is due to chance, i.e. the source is variable at only $60 \%$ confidence level which reject the hypothesis of variability. We have also made a mosaic of such four consecutive ScWs, this yielded to a source detection of $\sim 11 \sigma(22-60 \mathrm{keV})$. Given the good 
Table 3. List of single ScWs in revolutions n. 1628 and 1629 during which significant source detections (i.e. $\geq 5 \sigma$ ) where achivied in the energy band $22-60 \mathrm{keV}$.

\begin{tabular}{cccc}
\hline \hline $\begin{array}{c}\text { Telescope orbit } \\
(\text { n.) }\end{array}$ & $\begin{array}{c}\text { ScW } \\
(\mathrm{n} .)\end{array}$ & $\begin{array}{c}\text { Flux } \\
(\mathrm{mCrab})\end{array}$ & $\begin{array}{c}\text { Significance } \\
(\sigma)\end{array}$ \\
\hline 1628 & 30 & $66.5 \pm 10.2$ & 6.5 \\
1629 & 20 & $29.4 \pm 5.2$ & 5.6 \\
1629 & 21 & $22.8 \pm 4.2$ & 5.4 \\
1629 & 22 & $24.8 \pm 4.3$ & 5.8 \\
1629 & 23 & $28.5 \pm 5.1$ & 5.6 \\
1629 & 33 & $76.1 \pm 13.4$ & 5.7 \\
1629 & 35 & $47.6 \pm 9.2$ & 5.2 \\
\hline \hline
\end{tabular}

statistics, we extracted an IBIS/ISGRI spectrum which was best fit by a black body $\left(\chi_{\nu}^{2}=0.9,4\right.$ d.o.f., see Fig. 3$)$ with $\mathrm{kT}=8.6_{-1.2}^{+1.4} \mathrm{keV}$ and average $18-60 \mathrm{keV}(20-40 \mathrm{keV})$ flux of $\sim 6 \times 10^{-10} \mathrm{erg} \mathrm{cm}^{-2} \mathrm{~s}^{-1}\left(\sim 4 \times 10^{-10} \mathrm{erg} \mathrm{cm}^{-2} \mathrm{~s}^{-1}\right)$. We note that both a thermal bremsstrahlung $\left(\chi_{\nu}^{2}=0.6,4\right.$ d.o.f. $)$ and a power law $\left(\chi_{\nu}^{2}=0.75,4\right.$ d.o.f $)$ provided a good description of the spectrum as well, with best fit parameter values equal to $\mathrm{kT}=34_{-11}^{+23} \mathrm{keV}$ and $\Gamma=2.5 \pm 0.5$, respectively. All such best fit parameter values are consistent, within their uncertainties, with those obtained from the detection during revolution n. 1605 .

\subsubsection{IBIS/ISGRI refined position and upper limit}

In order to get the most refined IBIS/ISGRI position of the source, we made a mosaic summing up all the three revolutions 1605, 1628 and 1629. AX J1949.8+2534 was detected at $11.2 \sigma$ level $(22-60 \mathrm{keV})$ with a total on-source exposure of $\sim 50$ ks. Fig. 4 shows the corresponding significance map. The best position is $\mathrm{RA}=297^{\circ} .49$ and $\mathrm{Dec}=25^{\circ} .57$ with a $90 \%$ confidence error circle radius equal to $2^{\prime} .4$.

AX J1949.8+2534 is not listed in the latest published IBIS/ISGRI catalog (Bird et al. 2016) despite extensive $I N$ TEGRAL coverage of its sky region $(\sim 2 \mathrm{Ms}$ up to revolution n. 1000 considered in Bird et al. 2016) and this information can be used to infer an upper limit on its persistent hard $\mathrm{X}$-ray emission. By additionally considering the source exposure from our present dataset $(\sim 0.25 \mathrm{Ms})$, we can infer a $3 \sigma$ upper limit of $\sim 0.4 \mathrm{mCrab}$ or $3.2 \times 10^{-12} \mathrm{erg} \mathrm{cm}^{-2}$ $\mathrm{s}^{-1}(20-40 \mathrm{keV})$ for persistent emission. When assuming the source peak flux as measured by IBIS/ISGRI from the outburst reported in section 2.2.2, we can infer a dynamic range of $\geq 625$

\section{SOFT X-RAY OBSERVATIONS}

\subsection{Swift/XRT}

Following the two newly discovered IBIS/ISGRI detections of AX J1949.8+2534 reported here, we triggered a ToO observation of the sky region with the Swift satellite (Gehrels et al. 2004) with the main aims to i) refine the position of the source with a much higher accuracy; ii) perform a spectral analysis in soft X-rays for the first time. The observation was performed on 2016, April 25 (ID: 00034497001).

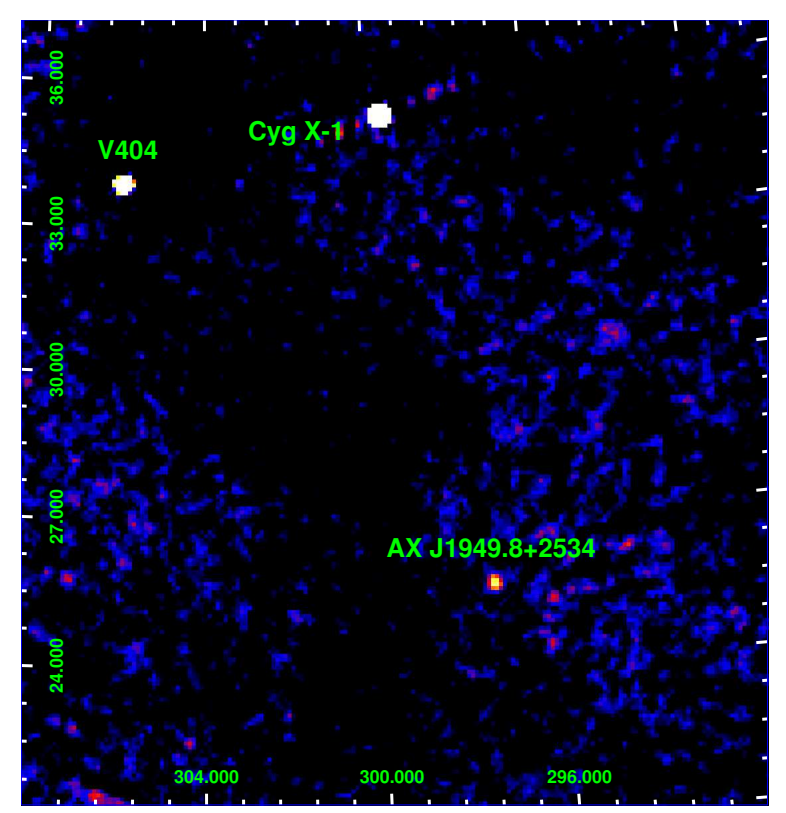

Figure 4. IBIS/ISGRI significance mosaic map (22-60 keV) obtained by summing revolutions $1605,1628,1629$. The source AX J1949.8+2534 is detected at $\sim 11 \sigma$ level.

Standard data reduction and analysis were perfomed using HEASOFT version 6.18 together with the most updated Swift/XRT calibration files. The XRT data were reprocessed using XRTPIPELINE (v0.13.2).

The Swift/XRT (PC) observation resulted in a net exposure time of $2,929 \mathrm{~s}$, and it detected a faint X-ray counterpart $(\sim 5.3 \sigma, 0.3-10 \mathrm{keV})$ within both the IBIS/ISGRI and $A S C A$ error circles (see Fig. 5). The best determined XRT position is at R.A. $(\mathrm{J} 2000)=19^{\mathrm{h}} 49^{\mathrm{m}} 55^{\mathrm{s}} .19$ Dec $(\mathrm{J} 2000)=$ $+25^{\circ} 33^{\prime} 57^{\prime \prime} .5$ with an error radius of $3^{\prime \prime} .7$ (90\% confidence) using the XRT-UVOT alignment and matching UVOT field sources to the USNO-B1 catalog (see Evans et al. 2009 and http:/www.swift.ac.uk/user_objects).

We then extracted counts from a circle with a radius of 20 pixels centered on the source position, together with a background from an annular region centered on the source position, with inner and outer radii of 30 and 60 pixels, repectively. The source net count rate in the energy range $0.3-10 \mathrm{keV}$ was $(8.08 \pm 1.71) \times 10^{-3}$ counts s$^{-1}$.

Given the low statistics, we rebinned the spectrum to 1 count bin $^{-1}$ and adopted Cash statistics (Cash 1979) in XSPEC. The Swift/XRT spectrum was well fitted by an absorbed power law (C-Stat $=20.8,24$ d.o.f.) where the absorption $\mathrm{N}_{\mathrm{H}}$ was fixed at $1.18 \times 10^{22} \mathrm{~cm}^{-2}$ (the total Galactic absorption in the source direction, Willingale et al. 2013). We obtained a photon index of $\Gamma=0.2 \pm 0.9$. The $0.3-10 \mathrm{keV}$ observed flux was $1.8 \times 10^{-12} \mathrm{erg} \mathrm{cm}^{-2} \mathrm{~s}^{-1}$ $\left(2 \times 10^{-12} \mathrm{erg} \mathrm{cm}^{-2} \mathrm{~s}^{-1}\right.$ corrected for the absorption $)$.

\subsection{Chandra}

The ACIS High Resolution Camera onboard Chandra (Weisskopf et al. 2000) observed AX J1949.8+2534 on 2008 Feb 08 for a total exposure time of $\sim 1.16 \mathrm{ks}$. This targeted observation was performed in the context of the ChIcAGO survey 
Table 4. List of NIR sources (as taken from the UKIDSS Galactic Plane Survey) located inside the 90\% confidence error circle (n. 1) and $95 \%$ confidence error circle (from n. 2 to n. 5 ) of AX 1949.8+2534. † source also reported in the 2MASS catalog as J19495543+2533599. The table lists their JHK magnitudes (lower limits are derived according to Lawrence et al. 2007), offset from the XRT coordinates, Q value (see section 4), inferred spectral type, reddening and distance

\begin{tabular}{|c|c|c|c|c|c|c|c|c|c|}
\hline n. & name & $\begin{array}{c}\mathrm{J} \\
(\mathrm{mag})\end{array}$ & $\begin{array}{c}\mathrm{H} \\
(\mathrm{mag})\end{array}$ & $\begin{array}{c}\mathrm{K} \\
(\mathrm{mag})\end{array}$ & offset & $\mathrm{Q}$ & Spectral Type & $\begin{array}{c}\mathrm{A}_{v} \\
(\mathrm{mag})\end{array}$ & $\begin{array}{c}\mathrm{d} \\
(\mathrm{kpc})\end{array}$ \\
\hline 1 & J194955.02+253354.6 & $>19.9$ & $>19.0$ & $17.788 \pm 0.142$ & $3.66^{\prime \prime}$ & & & & \\
\hline 2 & J194954.99+253400.2 & $17.094 \pm 0.015$ & $16.182 \pm 0.013$ & $15.743 \pm 0.022$ & $3.78^{\prime \prime}$ & 0.17 & late type & & \\
\hline 3 & $\mathrm{~J} 194955.12+253401.4$ & $14.889 \pm 0.003$ & $14.263 \pm 0.003$ & $13.902 \pm 0.005$ & $4.02^{\prime \prime}$ & 0.01 & B0V & 7.1 & 17.3 \\
\hline 4 & J194955.31+253353.8 & $17.102 \pm 0.015$ & $15.878 \pm 0.010$ & $15.285 \pm 0.015$ & $4.02^{\prime \prime}$ & 0.22 & $\mathrm{~K} 0 \mathrm{~V}$ & 4.4 & 1.3 \\
\hline $5 \dagger$ & J194955.42+253359.9 & $9.900 \pm 0.022$ & $9.071 \pm 0.016$ & $8.637 \pm 0.018$ & $4.02^{\prime \prime}$ & 0.09 & B0.5Ia & 7.2 & 8.8 \\
\hline
\end{tabular}

(Anderson et al. 2014), aimed at classifying a selected list of unidentified X-ray sources discovered during the $A S C A$ Galactic plane survey. No significant X-ray source was detected inside the entire field of view pertaining to this Chandra observation, the ChIcAGO survey team reports only a $\sim 2 \sigma$ excess (labelled as ChI 194951+2534_1 from Table 1 in Anderson et al. 2014) located at $1^{\prime} .6$ from the $A S C A$ position of AX J1949.8+2534. We used the count rate of ChI 194951+2534_1 in order to estimate with WEBPIMMS a $0.3-10 \mathrm{keV}$ observed (unabsorbed) $3 \sigma$ upper limit of $9.6 \times 10^{-13} \mathrm{erg} \mathrm{cm}^{-2} \mathrm{~s}^{-1}\left(1.05 \times 10^{-12} \mathrm{erg} \mathrm{cm}^{-2} \mathrm{~s}^{-1}\right)$ for AX J1949.8+2534. We assumed the same spectral model as from the Swift/ XRT observation. This is a factor of about 2 lower than the X-ray flux measured during the Swift/XRT detection.

\section{SEARCH FOR INFRARED, OPTICAL AND RADIO COUNTERPARTS}

The identification of lower energy counterparts represents a mandatory step in determining the nature of unidentified Galactic X-ray sources. To this aim, we obtained with Swift/XRT a $90 \%$ confidence source positional accuracy of $3^{\prime \prime} .7$ which significantly improves the previously available $A S C A$ uncertainty of $\sim 1^{\prime}$. This allowed us to perform for the first time a search for counterparts from radio to optical bands, by using all the available catalogs in the HEASARC database.

No catalogued radio and optical source is located within the arcsecond sized XRT error circle. In the optical $\mathrm{V}$ band, a lower limit of $\mathrm{V}>21$ can be inferred from the USNO survey limit as reported in Monet et al. (2003). Conversely, only one near infrared (NIR) source has been detected by the UKIDSS Galactic Plane Survey (Lucas et al. 2008) inside the $90 \%$ confidence $X R T$ error circle, such object is listed in Table 4 as n. 1. Fig. 6 shows both the XRT error circles at $90 \%$ and $95 \%$ confidence, superimposed on the $K$ band UKIDSS image. We note that source n. 1 is located at $3^{\prime \prime} .66$ distance from the XRT coordinates centroid, i.e. on the $90 \%$ confidence error circle (radius of $3^{\prime \prime} .7$, solid circle). It is very faint in the $K$ band (magnitude of $\sim 17.8$, as such it is not evident in the image) and it is undetected in the $J$ and $H$ bands. As a consequence we consider very unlikely the possibility that this extremely faint object could be a reliable NIR counterpart of AX J1949.8+2534. For the sake of completeness, in Fig. 6 we note that a few brighter infrared

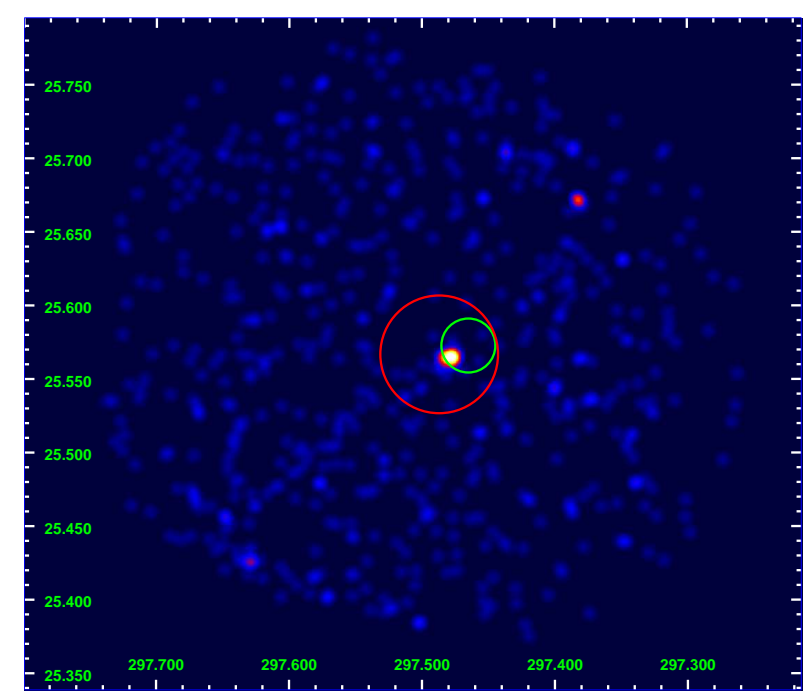

Figure 5. Swift/XRT image (0.3-10 keV) with superimposed the $A S C A$ (green) and IBIS/ISGRI (red) error circles having a radius of $1^{\prime}$ and $2^{\prime} .4$, respectively. AX J1949.8+2534 is detected inside both error circles at $\sim 5.3 \sigma$ level.

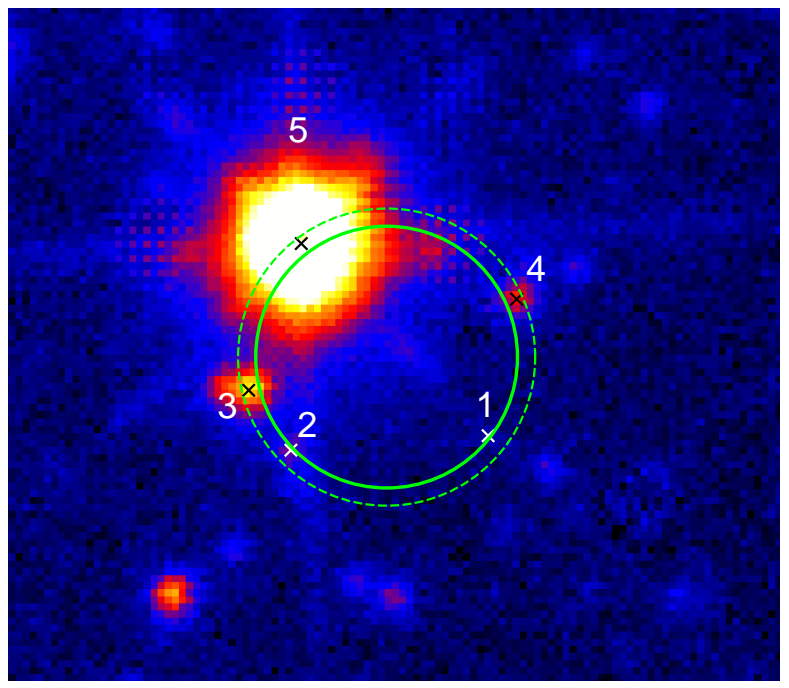

Figure 6. UKIDSS image in the $K$ band (as downloaded from the UKIDSS archive, http://wsa.roe.ac.uk) with superimposed the Swift/XRT error circles at $90 \%$ confidence (solid green, radius of $3^{\prime \prime} .7$ ) and $95 \%$ confidence (dashed green, radius of $4^{\prime \prime} .2$ ) 
Sguera et al.

sources are located at $\sim 4^{\prime \prime}$ distance from the $X R T$ centroid, i.e. slightly outside the $90 \%$ confidence error circle (radius of $3^{\prime \prime} .7$, solid circle) and well inside the $95 \%$ confidence $X R T$ error circle radius of $4^{\prime \prime} .2$ (dashed circle). Such objects are listed in Table 4 with numbers from 2 to 5 .

If we use the reddening-free NIR diagnostic $Q$ of Negueruela \& Schurch (2007) for the objects n. 2 and n. 4 in Table 4 , then we find that none of them has a $Q$ value typical of early-type stars (i.e. $\leq 0$ ). As a matter of fact, they show $Q$ values of 0.17 and 0.22 respectively, which are much more similar to those of intermediate or late type stars. For example, if we consider the brightest infrared object among the two (n. 4), then we find that it is compatible with being a star of spectral type $\mathrm{K} 0 \mathrm{~V}$ with a reddening of $\mathrm{A}_{v}=4.4 \mathrm{mag}$, located at a distance of $\sim 1.3 \mathrm{kpc}$. Its implied $V$ magnitude is $\sim 23.4$, which is consistent with not detecting it in the $\mathrm{V}$ band according to the USNO catalog. As for the brightest infrared object (n. 5), it is also reported in the 2MASS infrared source catalog (namely J19495543+2533599) with magnitudes equal to $J=9.900 \pm 0.022, H=9.071 \pm 0.016$ and $K=8.637 \pm 0.018$ as well as in the optical USNO-B1.0 cata$\log (1155-0421415)$ with magnitudes of $I=12.87, R 2=14.62$, $B 2=17.35$. Notably, its $Q$ value (0.09) is typical of early-type stars (Negueruela \& Schurch 2007). Moreover, by comparing its $K$ magnitude with the right panel of Fig. 1 in Reig \& Milonaki (2016) we note that it is located inside the box populated by blue supergiants. In fact, we found that the observed $K$ and $J$ magnitudes and the observed $J-K$ color are compatible with a B0.5Ia spectral type supergiant star, for a distance of $\sim 8.8 \mathrm{kpc}$ and a reddening of $\mathrm{A}_{v}=7.2 \mathrm{mag}$. Its implied apparent $V$ magnitude is $\sim 15$, which is consistent with the detection of the source as reported in the USNO catalog. Finally, also the infrared object n. 3 is characterized by a $Q$ value (0.01) typical of an early-type star. In this case, its observed NIR magnitudes are not compatible with being a supergiant star since this would require an extremely large distance ( $\sim 70 \mathrm{kpc}$, i.e. object outside the Galaxy) and an high extinction $\left(\mathrm{A}_{v}=6.7 \mathrm{mag}\right)$. On the other hand, it is more compatible with being a main sequence B0V spectral type star, for a distance of $\sim 17.3 \mathrm{kpc}$ and a reddening of $\mathrm{A}_{v}=7.1$ mag, the implied apparent $V$ magnitude is $\sim 19.3$, which is consistent with the detection of the source as reported in the USNO catalog with a B magnitude equal to $\sim 19$.

\section{DISCUSSION}

We have presented mainly IBIS/ISGRI results on newly discovered hard X-ray activity from the unidentified transient AX J1949.8+2534, the first ever emission to be reported above $20 \mathrm{keV}$. Hard X-ray outbursts have been detected twice, on November 2015 and January 2016, respectively. Furthermore, we point out that we have searched the entire currently available IBIS/ISGRI public data archive (revolution 25-1619, Paizis et al. 2013, 2017) for possible additional outbursts of AX J1949.8+2534. No detections have been found at $\mathrm{ScW}$ level above a significance value of $7 \sigma$ in both energy bands $22-50$ and $50-100 \mathrm{keV}$. The source exposure time obtained from the entire archive is of the order of $\sim 7 \mathrm{Ms}$. The inferred duty cycle is as low as $\sim 4 \%$.

We can use all the collected multiwavelength data to consider the possible nature of AX J1949.8+2534.

\subsection{Low Mass X-ray Binary or Cataclysmic Variable?}

As we noted before, two NIR objects, compatible with being late type spectral stars, are present within the $95 \%$ confidence XRT error circle (n. 2 and n. 4 in Table 4 and Fig. 6). In principle, this could suggest a Low Mass X-ray Binary nature (LMXB) or alternatively a Cataclysmic Variable one $(\mathrm{CV})$. However, in the following we show that both such scenarios suffer serious drawbacks when broad-band X-ray results are taken into account.

The LMXB hypothesis is incompatible with both the very hard X-ray spectrum measured by Swift/XRT (i.e. $\Gamma \sim 0.2)$ as well as with the particularly short duration of the hard X-ray activity detected by IBIS/ISGRI ( 1.5 days, in the only case when it was possible to firmly constrain the duration). In fact, transient LXMBs are know to display $\mathrm{X}$-ray outbursts whose duration is typically of the order of weeks/months, they are characterized by soft X-ray spectra below $10 \mathrm{keV}$ (due to disk black body emission). From Table 4, we note that the two NIR objects compatible with a late type nature (i. e. n. 2 and n. 4) are particularly weak. If we consider as possible counterpart the brightest among the two (n. 4) then we showed that it is compatible with a late type star located at $\sim 1.3 \mathrm{kpc}$. The corresponding average X-ray luminosities during the two hard X-ray outbursts detected by IBIS/ISGRI are of the order of $\sim 2 \times 10^{34} \mathrm{erg} \mathrm{s}^{-1}$. This is way too low if compared to typical luminous X-ray outbursts from LMXBs ( up to $\sim 10^{37-38} \mathrm{erg} \mathrm{s}^{-1}$ ). For the sake of completeness, we note that recently a growing number of LMXBs have been found to show outbursts reaching peak X-ray luminosity of only $\sim 10^{34-36} \mathrm{erg} \mathrm{s}^{-1}$ in the soft X-ray band 2-10 keV. They belong to a more general class of X-ray transients dubbed as Very Faint X-ray Transients (VFXTs), which are believed to be the the faintest known X-ray accretors (Degenaar \& Wijnands 2009, 2011). If we extrapolate the IBIS/ISGRI spectral shape of AX J1949.8+2534 in outbursts to the $2-10 \mathrm{keV}$ X-ray flux, then we obtain a $2-10 \mathrm{keV}$ X-ray luminosity (at $1.3 \mathrm{kpc}$ ) of the order of $\sim 2 \times 10^{33} \mathrm{erg} \mathrm{s}^{-1}$, i.e. lower than that typical of VFXTs. With all the above information at hand a LMXB nature for AX J1949.8+2534 seems to be not viable.

Alternatively, we are left with the CV hypothesis. In principle, both the Swift/XRT and IBIS/ISGRI spectral Xray characteristics are compatible with this scenario (Barlow et al. 2006, Landi et al. 2009). However, we note that to date all the CVs detected by both IBIS/ISGRI and Swift/BAT above $20 \mathrm{keV}$ are weak persistent hard X-ray sources with typical luminosities in the range $\sim 10^{32-34} \mathrm{erg} \mathrm{s}^{-1}$ (Barlow et al. 2006, Revnivtsev et al. 2008, Brunschweiger et al. 2009). No CV has never been detected as a transient hard X-ray source. This is completely at odds with the short transient behavior of AX J1949.8+2534 as observed by IBIS/ISGRI. Moreover, if we consider the brightest NIR object compatible with a late type nature (n. 4), then it is compatible with a late type M5V main sequence star (which is typical of CVs, Smith et al. 1998) located at $\sim 230$ pc. Consequently, the IBIS/ISGRI upper limit on the persistent hard X-ray emission of AX J1949.8+2534 would translate into a $20-40 \mathrm{keV}$ luminosity of $\leq 2 \times 10^{31} \mathrm{erg} \mathrm{s}^{-1}$, i.e. significantly lower than typical measurements obtained to date with both IBIS/ISGRI and Swift/BAT. The same holds for 
the Swift/XRT detection whose soft X-ray flux would translates into a luminosity of $\sim 1.2 \times 10^{31} \mathrm{erg} \mathrm{s}^{-1}$. All the above results point to largely disfavor the $\mathrm{CV}$ interpretation for AX J1949.8+2534.

\subsection{High Mass X-ray Binary}

The location of the source on the Galactic plane $\left(\mathrm{b} \sim-0.3^{\circ}\right)$ and both Swift/XRT and IBIS/ISGRI spectral characteristics (especially the hardness of the soft X-ray spectrum, i.e. $\Gamma \sim 0.2)$ are fully compatible with a High Mass X-ray Binary nature (HMXB). In particular, the hard X-ray transient behavior (e.g. dynamic range $\geq 620$ and duration of $\sim 1.5$ days and $\geq 4$ days from the two IBIS/ISGRI detected activities, respectively) could be typical of the Be HMXB class. In addition we note that mentioned X-ray characteristics are compatible as well with a Supergiant Fast X-ray Transients nature (SFXTs, Sguera et al. 2008), which are a newly discovered class of HMXBs (Sguera et al. 2005, 2006, Negueruela et al. 2005)

Although classical SFXTs usually display above 20 $\mathrm{keV}$ hard X-ray outbursts lasting much less than a day, a few other SFXTs are known to show unusually longer hard X-ray activity, exceptionally lasting several days (e.g. IGR J18483-0311, Sguera et al. 2015; IGR J17354-3255, Sguera et al. 2011), i.e. comparable to the duration of the hard X-ray activity detected from AX J1949.8+2534.

We must note that, in principle, this proposed HMXB interpretation could suffer some drawbacks when NIR data are combined with the $90 \%$ confidence $X R T$ positional uncertainty. In fact, searching within the latter, we pinpointed only one very faint NIR object (n. 1 in Table 4), whose magnitudes and colors are not compatible with being an early type spectral star, i.e. at odds with an HMXB nature. However, slightly outside the $90 \%$ confidence XRT error circle (radius of $3^{\prime \prime} .7$ ) we note the presence of two bright NIR sources (n. 3 and n. 5) located at $4^{\prime \prime}$ distance from the XRT centroid, i.e. well inside the $95 \%$ confidence $X R T$ positional uncertainty (radius of $4^{\prime \prime} .2$ ). Both have observed $K$ and $J$ magnitudes and $J-K$ color compatible with an early type nature.

Specifically, the characteristics of the NIR object n. 3 are compatible with a main sequence early type spectral star (B0V), supporting a Be HMXB scenario. If we consider its calculated distance of $\sim 17.3 \mathrm{kpc}$, then the two hard X-ray outbursts detected by IBIS/ISGRI from AX J1949.8+2534 would have a $18-60 \mathrm{keV}$ average (peak) luminosity of $4 \times 10^{36}$ erg s$^{-1}\left(2 \times 10^{37} \mathrm{erg} \mathrm{s}^{-1}\right)$, both such values are typical of periodic type I X-ray outbursts from Be HMXBs. As for the soft X-ray band, the measured Swift/XRT flux translates into a luminosity of $\sim 6 \times 10^{34} \mathrm{erg} \mathrm{s}^{-1}$. Such value is not representative of the X-ray quiescence of Be HMXBs which is usually much lower. Conversely, it could be more typical of quasi-persistent Be HMXBs which are a small subclass characterized by a persistent low X-ray luminosity of $\sim 10^{34} \mathrm{erg} \mathrm{s}^{-1}$, varying by up to a factor of $\sim 10$ at most, hence not displaying type I or II outburst behavior (see Reig \& Roche 1999). We note that this latter characteristic is at odds with the bright outburst behavior detected by IBIS/ISGRI from AX J1949.8+2534, as such it represents a significant drawback for the Be HMXB scenario.

As for the association with the brightest NIR object $n$.
5, their characteristics are fully compatible with being an early type spectral star of supergiant nature (B0.5Ia). This would fully support an SFXT scenario. We are aware that, in principle, to extend the search for NIR counterparts outside the canonical $90 \%$ XRT positional uncertainty could eventually be a dangerous approach, because of the possibility that unrelated NIR sources could be included and mistakenly assumed as counterparts. Bearing this in mind, we took into account the possibility that such an association could be simply a chance coincidence and accordingly calculated the probability of a random association with an infrared source having magnitude $K \leq 8.6$ as given by $P=1-\mathrm{e}^{-\pi \rho d^{2}}$ (where $\mathrm{d}$ is the distance between the XRT centroid and the associated NIR candidate, and $\rho$ is the local spatial density of NIR sources computed in an area of a few degree radius around the candidate). We estimated a probability of $0.1 \%$, i.e. 0.001 chance coincidence is expected. Such particularly low probability strongly supports a real physical association, hence the viable possibility that this infrared object is a reliable counterpart of the unidentified X-ray source AX J1949.8+2534. If we consider the calculated distance of $\sim 8.8 \mathrm{kpc}$ in the case of a B0.5Ia spectral type supergiant for the proposed NIR counterpart, then the two hard X-ray outbursts detected by IBIS/ISGRI from AX J1949.8+2534 would have a $18-60 \mathrm{keV}$ average (peak) luminosity of $1.1 \times 10^{36} \mathrm{erg} \mathrm{s}^{-1}$ $\left(5.5 \times 10^{36} \mathrm{erg} \mathrm{s}^{-1}\right)$. From archival INTEGRAL/IBIS observations, we placed a $3 \sigma$ upper limit of $\sim 3 \times 10^{34} \mathrm{erg} \mathrm{s}^{-1}$ for the persistent hard X-ray luminosity. Both such values are very similar to those of known confirmed SFXTs. As for the soft X-ray band, the measured Swift/XRT flux translates into a luminosity of $\sim 2 \times 10^{34} \mathrm{erg} \mathrm{s}^{-1}$, which is fully compatible with the so-called intermediate intensity X-ray state during which typical SFXTs spend the majority of their time (Sidoli et al. 2008). In this context, we note that the prolongated duration of the hard X-ray activity discovered from AX J1949.8+2534 ( 1.5 and $\geq 4$ days, respectively) is at odds with the much shorter durations typically marking outbursts from classical SFXTs above $20 \mathrm{keV}$ (i.e. a few hours). A similar characteristic was previously reported only for a few other SFXT sources. Our new findings on AX J1949.8+2534 could strengthen the idea that unusually long hard X-ray outbursts would not be particularly exceptional among the class of SFXTs.

\section{CONCLUSIONS}

We reported on the IBIS/ISGRI discovery of two new hard X-ray outbursts from the unidentified transient AX J1949.8+2534, the first ever emission to be detected above $20 \mathrm{keV}$. A follow-up observation of the sky region with the Swift satellite allowed for the first time to perform a soft X-ray spectral analysis as well as significantly improve the positional uncertainty to arcsecond size. This permitted us to pinpoint two bright infrared sources as most likely candidate counterparts. Both are compatible with being early type spectral stars hence supporting an HMXB nature, specifically an SFXT (more viable) or alternatively a Be HMXB. Further detailed NIR or optical spectroscopy is mandatory to confirm their putative supergiant and Be nature, respectively. Unfortunately, we can go no further on this issue because the current $\mathrm{X}$-ray positional uncer- 
tainty $\left(3^{\prime \prime} .7\right)$ prevents us from unambiguously pinpointing the correct single NIR counterpart. Additional X-ray observations of AX J1949.8+2534 using Chandra, for example, are strongly needed in order to achieve a finer position with an associated smaller error circle.

\section{ACKNOWLEDGMENTS}

We thank the anonymous referee for useful comments which helped us to improve the quality of this paper. We thank the Swift team, the PI, the duty scientists and science planners for making the two ToO observations reported here possible. We acknowledge financial support from the Italian Space Agency via INTEGRAL ASI/INAF agreement n. 2013-025.R.1, and the grant from PRIN-INAF 2014, "Towards a unified picture of accretion in High Mass X-Ray Binaries". This work has made use of the INTEGRAL archive developed at INAF-IASF Milano (http://www.iasfmilano.inaf.it/ ada/GOLIA.html). This research has made use of data and/or software provided by the High Energy Astrophysics Science Archive Research Center (HEASARC), which is a service of the Astrophysics Science Division at NASA/GSFC and the High Energy Astrophysics Division of the Smithsonian Astrophysical Observatory.

\section{REFERENCES}

Anderson, G. E., Gaensler, B. M., Kaplan, D. L., et al. 2014, ApJS, 212, 13

Barlow, E. J., Knigge, C., Bird, A. J., et al. 2006, MNRAS, 372, 224

Bird, A. J., Bazzano, A., Malizia, A., et al. 2016, ApJS, 223, 15

Brunschweiger, J., Greiner, J., Ajello, M., \& Osborne, J. 2009, A\&A, 496, 121

Cash, W. 1979, ApJ, 228, 939

Degenaar, N., \& Wijnands, R. 2009, A\&A, 495, 547

Degenaar, N., \& Wijnands, R. 2011, A\&A, 524, A69

Evans, P. A., Beardmore, A. P., Page, K. L., et al. 2009, MNRAS, 397,1177

Gehrels, N., Chincarini, G., Giommi, P., et al. 2004, ApJ, 611, 1005

Heise, J., in't Zand, J.J.M., Smith, M.J.S., et al. 1999, Ap. Lett. Comm., 38, 297

Krivonos R., Revnivtsev, M., Tsygankov, S., et al. 2010, A\&A, 519, 107

Landi, R., Bassani, L., Dean, A. J., et al. 2009, MNRAS, 392, 630

Lawrence et al., 2007, MNRAS, 379,1599

Lebrun F., et al., A\&A 2003, 411, 141

Lucas, P. W., Hoare, M. G., Longmore, A., et al. 2008, MNRAS, 391,136

Lund, N., Budtz-Jorgensen, C., Westergaard, N. J., et al. 2003, A\&A, 411L, 231

Monet et al. 2003, AJ, 125, 984

Negueruela I., Smith D., Reig P., et al. 2006, ESA SP-604: X-ray Universe 2005, Wilson A. editor, 2006, 165

Negueruela, I., \& Schurch, M.P.E. 2007, A\&A, 461, 631

Paizis, A., Mereghetti, S., Gotz, D., et al. 2013, Astron. Comput., 1,33

Paizis, A., et al. 2017, 11th INTEGRAL Conference Gamma-Ray Astrophysics in Multi-Wavelength Perspective Proceedings, submitted

Reig, P., \& Roche, P., 1999, MNRAS, 306, 100

Reig, P., \& Milonaki, F. 2016, A\&A, 594, A45
Revnivtsev, M., Sazonov, S., Krivonos, R., et al. 2008, A\&A, 489, 1121

Sguera, V., Barlow, E. J., Bird, A. J., et al. 2005, A\&A, 444, 221

Sguera, V., Bazzano, A., Bird, A. J., et al. 2006, ApJ, 646, 452

Sguera, V., Bassani, L., Landi, R., et al. 2008, A\&A, 487, 619

Sguera, V., Drave, S. P., Bird, A. J., et al. 2011, MNRAS, 417, 573

Sguera, V., Bazzano, A., Sidoli, L., 2015, ATel 8250

Sguera, V., Sidoli, L., Bird, A. J., et al. 2015, MNRAS, 449, 1228

Sidoli, L., Romano, P., Mangano, V., et al. 2008, ApJ, 687, 1230

Smith, D. A., Dhillon, V. S., 1998, MNRAS, 301, 767

Sugizaki, M., Mitsuda, K., Kaneda, H., et al., 2001, ApJS, 134, 77

Ubertini P., et al., 2003, A\&A, 411, L131

Weisskopf, M. C., Tananbaum, H. D., Van Speybroeck, L. P., et al. 2000, SPIE, 4012, 2

Willingale, R., Starling, R. L. C., Beardmore, A. P., et al. 2013, MNRAS, 431, 394

Winkler C., et al., 2003, A\&A, 411, L1 